these dynamics. In mammals, all these issues are further confused by the existence of multiple genes and/or splice variants for several of the proteins in these complexes, including Eps8, Abi1 and WAVE. Clearly, a detailed understanding of Eps8 function will require extensive further investigation, but some important initial steps on this path have been taken here.
1. Rafelski, S. M. \& Theriot, J. A. Annu. Rev. Biochem. 73, 209-239 (2004).

2. Wear, M. A. \& Cooper, J. A. Trends Biochem. Sci. 29, 418-428 (2004)

3. Krause, M., Dent, E. W., Bear, J. E., Loureiro, J. J. \& Gertler, F. B. Annu. Rev. Cell Dev. Biol. 19, 541-564 (2003).

4. Zigmond, S. H. Curr. Opin. Cell Biol. 16, 99-105 (2004).

5. Disanzo et al. Nature Cell Biol. 6, 1180-1188 (2004).

6. Croce et al. Nature Cell Biol. 6, 1173-1179 (2004).

7. Di Fiore, P. P. \& Scita, G. Int. J. Biochem. Cell Biol. 34 1178-1183 (2002).
8. Scita, G. et al. J. Cell Biol. 154, 1031-1044 (2001).

9. Offenhauser, N. et al. Mol. Biol. Cell 15, 91-98 (2004)

10. Scita, G. et al. Nature 401, 290-293 (1999).

11. Eden, S., Rohatgi, R., Podtelejnikov, A. V., Mann, M. \& Kirschner, M. W. Nature 418, 790-793 (2002).

12. Gautreau, A. et al. Proc. Natl Acad. Sci. USA 101, 43794383 (2004).

13. Steffen, A. et al. EMBO J. 23, 749-759 (2004).

14. Innocenti, M. et al. Nature Cell Biol. 6, 319-327 (2004).

15. Suetsugu, S., Miki, H. \& Takenawa, T. Cell Motil. Cytoskeleton 51, 113-122 (2002)

\title{
PINOID pinpoints auxin
}

Auxin functions as an endogenous signal whose distribution controls plant patterning and adaptation to the changing environment. Its efflux is controlled by the PIN-FORMED (PIN) family of proteins, and Friml et al. now report in Science $(306,862-865 ; 2004)$ that PIN localization is regulated through the action of the serine/threonine kinase, PINOID (PID).

PIN proteins are asymmetrically localized determinants at the plasma membrane that direct the auxin flow. This in turn establishes the gradient that controls a variety of developmental processes, including apical patterning. Friml et al. used a combination of gainand loss-of-function approaches to demonstrate that PID regulates auxin transport by specifically controlling the apicobasal polarity of PIN proteins.

When the authors crossed a transgenic line that constitutively expressed PID onto pin-mutant backgrounds, root collapse was significantly delayed, with a concomitant accumulation of auxin in the root. More importantly, they observed that overexpression of PID shifted the polarity of PIN from basal to apical - in both embryonic and adult cells - without affecting PIN's expression level or stability. This shift in PIN localization required the kinase domain of PID and was dose-dependent. The authors concluded that overexpression of PID causes PIN to move apically, which reverses the auxin flow and causes developmental defects, such as agravitropy and mis-specification of the root pole.

Both pin 1 and pid loss-of-function mutants form needle-like inflorescences that develop only few flowers and bracks. To test whether endogenous PID was necessary for the proper localization of PIN1, the authors examined the distribution of PIN1 fused to green fluorescent protein (PIN1-GFP) when pid is lost. They showed that in epidermal cells of the shoot apex, where PIN1 is normally localized at the apical side, PIN1-GFP was now localized basally. From this they inferred that the basal localization of PIN1 in the absence of PID inhibits the apical accumulation of auxin that is essential for aerial organ specification.
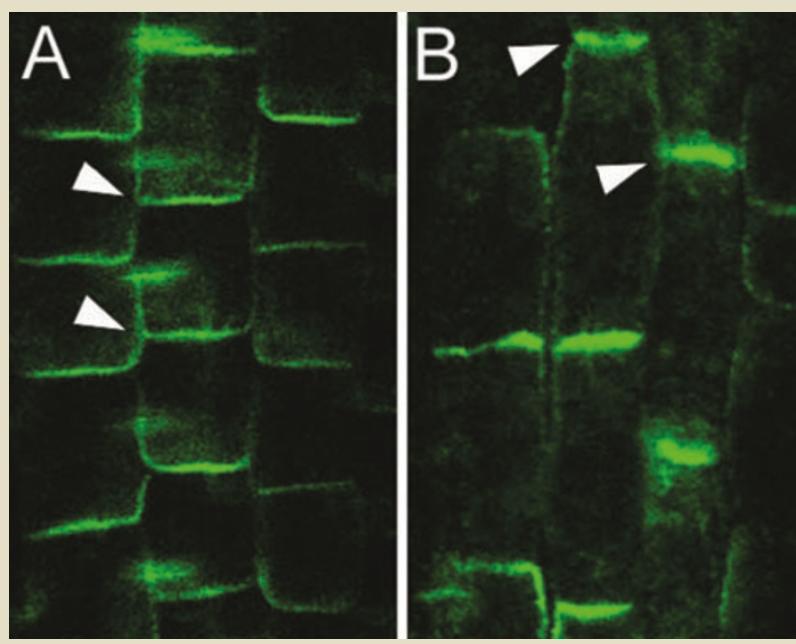

PIN1 (green) polarity in the root tip shifts from basal to apical in response to ectopic PID expression. (A) Wild-type, (B) PID overexpression. Image reproduced from Friml et al. Science 306, 862-865 (2004) with permission from the American Academy of Sciences (C) (2004).

GNOM, a guanine nucleotide-exchange factor that acts on ADPribosylation factor, has been implicated in regulating the transport of PIN between the endosome and the plasma membrane. In gnom mutants, however, PIN becomes randomly distributed rather than specifically mis-localized as in pid mutants. Thus, Friml et al. seem to have identified a specific determinant of PIN polarization, and propose that PID functions as a binary switch, with sub-threshold levels resulting in basal distribution of PIN and above-threshold levels targeting PIN apically. It remains to be determined whether PIN is a direct target of PID, and how PID is itself regulated. One possibility is that auxin could form a positive feedback loop, as has already been suggested by the authors; or GNOM could be involved in steering PID activity.

Myrto Raftopoulou 